\title{
Targeted Protein Degradation with Small Molecules: How PROTACs Work ${ }^{+}$
}

\author{
Alessio Ciulli \\ School of Life Sciences, University of Dundee, Dow Street, Dundee, Scotland DD1 5EH, UK; \\ a.ciulli@dundee.ac.uk \\ + Presented at the 2nd Molecules Medicinal Chemistry Symposium (MMCS): Facing Novel Challenges in \\ Drug Discovery, Barcelona, Spain, 15-17 May 2019.
}

Published: 16 December 2019

\begin{abstract}
Bivalent degrader molecules (also termed PROTACs) target proteins for degradation through recruitment to E3 ligases. PROTACs are a revolutionary new modality class with therapeutic potential. Formation of a ternary complex between the degrader, the ligase, and the target leads to tagging by ubiquitination and proteasomal degradation of the target protein. In 2015, we disclosed MZ1, a potent degrader made of a ligand we had previously discovered for the E3 ligase von Hippel-Lindau (VHL), and a pan-selective ligand for the BET proteins Brd2, Brd3, and Brd4. We made the unexpected but fascinating observation that MZ1 induces preferential degradation of Brd4 over Brd2 and Brd3-despite engaging BET proteins with the same binary affinity. This demonstrated a now well-established feature of PROTACs: They can achieve a narrower degradation profile in spite of broad target engagement. Our co-crystal structure of a PROTAC ternary complex (VHL:MZ1:Brd4) illuminated the role of cooperative molecular recognition inducing de novo contacts to form a stable ternary. Our work is revealing the structural basis and guiding principles of PROTAC degradation selectivity and mode of action.
\end{abstract}

(C) 2019 by the authors. Licensee MDPI, Basel, Switzerland. This article is an open access article distributed under the terms and conditions of the Creative Commons Attribution (CC BY) license (http://creativecommons.org/licenses/by/4.0/). 